\title{
Estimated excess acute-care length of stay and extra cost of testing-based versus symptom-based isolation strategies among veterans hospitalized with coronavirus disease 2019 (COVID-19) discharging to a congregate setting
}

\author{
Chenwei Wu MD ${ }^{1,2}$ (D), Susan Glass MD ${ }^{1,2}$, Sandra Demars MD ${ }^{1,2}$, Luis G. Tulloch-Palomino MD ${ }^{1,3, a}$ and \\ Pandora L. Wander MD, MS ${ }^{1,2, a}$ \\ ${ }^{1}$ Hospital and Specialty Medicine, Veterans Affairs Puget Sound Health Care System, Seattle, Washington, ${ }^{2}$ Division of General Internal Medicine, Department of \\ Medicine, University of Washington School of Medicine, Seattle, Washington and ${ }^{3}$ Division of Allergy and Infectious Diseases, Department of Medicine, University \\ of Washington School of Medicine, Seattle, Washington
}

During the coronavirus disease 2019 (COVID-19) pandemic, hospitals worldwide have experienced capacity shortfalls because of high case volumes compounded by prolonged length of stay (LOS). ${ }^{1}$ Although illness severity plays a major role in prolonging hospitalization, asymptomatic or recovered patients could potentially recuperate in congregate settings but may be prevented from discharging due to persistent yet clinically inconsequential viral shedding. ${ }^{1-7}$ Because COVID-19 transmission is unlikely $>10$ days after symptom onset, ${ }^{3-5}$ the US Centers for Disease Control and Prevention (CDC) in August 2020 recommended symptom-based rather than testing-based isolation measures. ${ }^{7}$ Implications of these recommendations for hospital operations remain unclear. We studied veterans hospitalized with COVID-19 who required discharge to a congregate setting (rehabilitation center, skilled nursing facility, public shelter, or supervised long-term care) to determine the impact of a symptom, rather than testing-based isolation strategy on acute care LOS and cost.

\section{Methods}

We included adults diagnosed with COVID-19 by nasopharyngeal or mid-turbinate specimen reverse-transcriptase polymerase chain reaction assay (RT-PCR) using the Cepheid Xpert Xpress test (Cepheid, Sunnyvale CA) or the Abbott M2000 test (Abbott Laboratories, Abbott Park, IL) between March 2 and June 2, 2020, within the Veterans Affairs Puget Sound Health Care System (VAPSHCS), a large, integrated, federal healthcare network

Author for correspondence: Chenwei Wu, E-mail: chenwei.wu2@va.gov

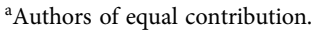

Cite this article: Wu C, et al. (2021). Estimated excess acute-care length of stay and extra cost of testing-based versus symptom-based isolation strategies among veterans hospitalized with coronavirus disease 2019 (COVID-19) discharging to a congregate setting. Infection Control \& Hospital Epidemiology, 42: 356-358, https://doi.org/ $10.1017 /$ ice.2020.1295 serving western Washington State. Discharge to congregate setting was contingent on 2 consecutive negative RT-PCR tests $>24$ hours apart.

Trained physician reviewers (C.W., L.G.T., S.G., S.D., and P.L.W.) collected the following data from the electronic health record: hospital admission, discharge, and testing dates. Severe illness and immunocompromised status were defined using CDC criteria (Table 1). ${ }^{7}$ The discharge eligibility date was established retrospectively according to CDC guidance (eg, afebrile, symptomatic improvement, and $>10$ days from onset or first positive RT-PCR for mild or asymptomatic disease if immunocompetent and $>20$ days if illness was severe or immunocompromised) or upon resolution of other conditions requiring hospitalization, whichever occurred later. ${ }^{7}$ All charts underwent independent review by a second, blinded investigator; in cases of disagreement, the later discharge eligibility date was used.

Excess acute-care LOS was defined as the difference between the true discharge date and the discharge eligibility date. Excess cost of care was determined by multiplying the "excess" fraction of a patient's stay by the total acute-care cost reported by the Veterans Health Administration (VHA) Allocation Resource Center (ARC). ARC costs are based on the managerial cost accounting system used widely in VHA cost-effectiveness research, adjusted for administrative overhead and special fees. ${ }^{8-10}$ Emergency department and intensive care costs were excluded. This study was approved by the VAPSHCS Institutional Review Board. The requirement for informed consent was waived.

\section{Results}

Overall, 70 veterans were diagnosed with COVID-19 during the study period and $29(41.4 \%)$ required hospitalization. Among them, 11 (37.9\% of hospitalized cases) were discharged to a congregate setting and were included in this analysis. Furthermore, 10 (90.9\%) were admitted to VAPSHCS; 1 was admitted to a community hospital and lacked cost information. All were male, with median age of 74 years (range, $68-100)$. In addition, $9(81.8 \%$ ) had

(c) The Author(s), 2020. Published by Cambridge University Press on behalf of The Society for Healthcare Epidemiology of America. This is an Open Access article, distributed under the terms of the Creative Commons Attribution licence (http://creativecommons.org/licenses/by/4.0/), which permits unrestricted re-use, distribution, and reproduction in any medium, provided the original work is properly cited. 


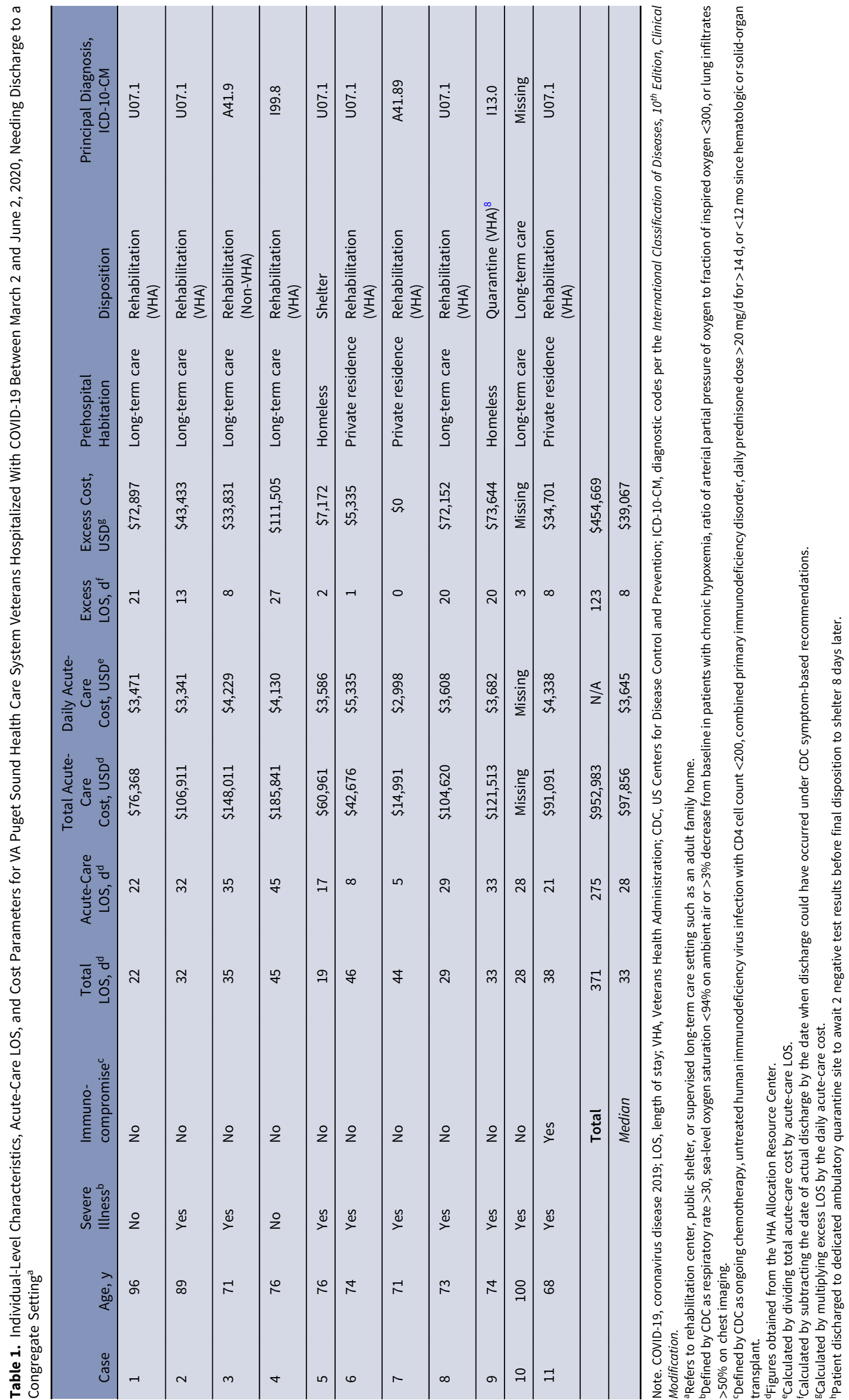


severe illness and 1 (9.1\%) was immunocompromised due to solidorgan transplantation (Table 1$)$.

Among this cohort, 7 patients $(63.6 \%)$ were discharged to a VHA-managed rehabilitation unit. One individual originally destined for this setting improved sufficiently to relocate to a VHA ambulatory quarantine site. One patient was discharged to a non-VHA rehabilitation center, 1 was discharged directly to shelter, and 1 was discharged to his prior adult family home (Table 1).

Testing-based isolation practices generated a cumulative 123 excess bed days of care and \$454,669 in additional cost. Median excess acute-care LOS was 8 days (range, 0-27 days). Among 10 patients with ARC financial data, median excess cost was $\$ 39,067$ (range, $\$ 0-\$ 111,505$ ) and cost per additional inpatient day was $\$ 3,645$ (range, $\$ 2,998-\$ 5,335$ ). In total, 275 bed days and $\$ 952,983$ were spent in acute care, of which $>40 \%$ (123 days and $\$ 454,669)$ could have been avoided using new symptom-based recommendations (Table 1 ).

\section{Discussion}

In our analysis, postsymptomatic inpatients with COVID-19 needing discharge to a congregate setting remained hospitalized a median 8 days longer and generated nearly $\$ 40,000$ in additional cost per person under a testing-based rather than symptom-based isolation strategy. Symptom-based precautions could have reduced the total LOS and cost by $>40 \%$. These calculations were made using conservative interpretations of the discharge eligibility date, which may result in underestimates. To our knowledge, this is the first analysis of operational consequences for facilities following a testing-based rather than a symptom-based isolation strategy. Our findings suggest nontrivial benefits of the latter, both in LOS reductions as well as cost savings.

Moreover, 8 of the 11 patients (72.3\%) discharged to the VHA, rather than privately operated postacute care settings, including 1 to a specialized VHA quarantine site. Had ambulatory quarantine not been available, an additional 8 inpatient days and $\$ 26,600$ in cost would have accrued prior to achieving testing-based clearance. The existence of VHA-managed rehabilitation units and dedicated quarantine sites demonstrates a degree of operational flexibility that may not be shared by more fragmented community hospitals and their post-acute care partners. These system-level differences raise the possibility that the LOS and cost differential for a testingbased versus symptom-based isolation strategy may be amplified in the private sector.

The strengths of our study include the incorporation of true per-patient costs and chart review performed by trained clinicians blinded to those costs. Limitations include small sample size and limited generalizability.

These preliminary results suggest healthcare administrators and governmental authorities should act quickly to translate symptom-based isolation strategies into practice. They also stress the importance of establishing clinically significant transmission criteria, rather than relying on highly sensitive molecular assays alone, to inform isolation guidelines in future outbreaks.

Acknowledgments. Views, opinions, and endorsements expressed in this manuscript do not reflect those of the Department of Veterans' Affairs.

Financial support. No financial support was provided relevant to this article.

Conflicts of interest. All authors report no conflicts of interest relevant to this article.

\section{References}

1. Rees EM, Nightingale ES, Jafari Y, et al. COVID-19 length of hospital stay: a systematic review and data synthesis. BMC Med 2020;18:270-292.

2. Argenziano MG, Bruce SL, Slater CL, et al. Characterization and clinical course of 1,000 patients with coronavirus disease 2019 in New York: retrospective case series. BMJ 2020;369:m1996.

3. Wölfel R, Corman VM, Guggemos W, et al. Virological assessment of hospitalized patients with COVID-2019. Nature 2020;581:465-469.

4. Bullard J, Dust K, Funk D, et al. Predicting infectious SARS-CoV-2 from diagnostic samples. Clin Infect Dis 2020. doi: 10.1093/cid/ciaa638.

5. Cheng HY, Jian SW, Liu DP, et al. Contact tracing assessment of COVID-19 transmission dynamics in Taiwan and risk at different exposure periods before and after symptom onset. JAMA Intern Med 2020;180:1156-1163.

6. Gombar S, Chang M, Hogan CA, et al. Persistent detection of SARS-CoV-2 RNA in patients and healthcare workers with COVID-19. J Clin Virol 2020;129:104477.

7. US Department of Health \& Human Services. Discontinuation of transmission-based precautions and disposition of patients with COVID-19 in healthcare settings (interim guidance). Centers for Disease Control and Prevention website. https://www.cdc.gov/coronavirus/2019-ncov/hcp/ disposition-hospitalized-patients.html. Updated August 10, 2020. Accessed September 9, 2020.

8. Barnett PG. Determination of VA healthcare costs. Med Care Res Rev 2003;60:124S-141S.

9. Nelson RE, Hicken B, Vanneman M, Liu CF, Rupper R. The impact of a change in the price of VA health care on utilization of VA and Medicare services. Med Care 2018;56:569-576.

10. Allocation Resource Center. ARC patient costing process. US Department of Veterans' Affairs website. http://vaww.arc.med.va.gov/references/ ARC_Patient_Costing_Process_sept2018.htm. Updated September 2018. Accessed August 15, 2020 